Review Article

\title{
Impaired GABA Neural Circuits Are Critical for Fragile X Syndrome
}

\author{
Fei Gao, ${ }^{1}$ Lijun Qi, ${ }^{1}$ Zhongzhen Yang, ${ }^{1}$ Tao Yang, ${ }^{2}$ Yan Zhang, ${ }^{1}$ Hui Xu $\mathbb{D},{ }^{3}$ \\ and Huan Zhao iD ${ }^{1,3}$ \\ ${ }^{1}$ Department of Anesthesiology, Heze Municipal Hospital, Heze, 274031 Shandong, China \\ ${ }^{2}$ Department of Pain Treatment, Tangdu Hospital, Fourth Military Medical University, Xi'an 710038, China \\ ${ }^{3}$ Department of Neurobiology and Collaborative Innovation Center for Brain Science, School of Basic Medicine, Fourth Military \\ Medical University, Xi'an 710032, China
}

Correspondence should be addressed to Hui Xu; xubz@fmmu.edu.cn and Huan Zhao; zhaohuanjiyi1@163.com

Received 8 June 2018; Accepted 17 September 2018; Published 3 October 2018

Guest Editor: Jolanta Dorszewska

Copyright ( 2018 Fei Gao et al. This is an open access article distributed under the Creative Commons Attribution License, which permits unrestricted use, distribution, and reproduction in any medium, provided the original work is properly cited.

Fragile X syndrome (FXS) is an inheritable neuropsychological disease caused by silence of the fmr1 gene and the deficiency of Fragile X mental retardation protein (FMRP). Patients present neuronal alterations that lead to severe intellectual disability and altered sleep rhythms. However, the neural circuit mechanisms underlying FXS remain unclear. Previous studies have suggested that metabolic glutamate and gamma-aminobutyric acid (GABA) receptors/circuits are two counter-balanced factors involved in FXS pathophysiology. More and more studies demonstrated that attenuated GABAergic circuits in the absence of FMRP are critical for abnormal progression of FXS. Here, we reviewed the changes of GABA neural circuits that were attributed to intellectual-deficient FXS, from several aspects including deregulated GABA metabolism, decreased expressions of GABA receptor subunits, and impaired GABAergic neural circuits. Furthermore, the activities of GABA neural circuits are modulated by circadian rhythm of FMRP metabolism and reviewed the abnormal condition of FXS mice or patients.

\section{Introduction}

Fragile $\mathrm{X}$ mental retardation protein (FMRP) is widely expressed in neurons and glia in the brain and acts as an "interactor" regulating ribosome stalling, translational control, and synaptic plasticity in brain circuits [1-3]. FMRP contributes to cognition, emotions, and memory through the referred "interactor" role as well. Fragile X syndrome (FXS) patients are deficient of FMRP due to fmrl gene silence caused by a CGG trinucleotide amplification on Xq27.3 in the $5^{\prime}$-UTR on chromosome [4]. According to CGG trinucleotide expansion and clinical symptoms, FM allele mutationrelated syndromes could be divided into FXS ( $>200$ repeats) and FXTAS (55-199 repeats) during early diagnosis of FXS. For example, methylation-specific quantitative melt analysis (MS-QMA), respectively, identified methylation mosaicism in an additional $15 \%$ and $11 \%$ of patients in the Chilean and Australian reports, suggesting the presence of a cryptic FM [5, 6]. Other methods include a variety of polymerase chain reaction (PCR) techniques, such as high polymorphism markers for preimplantation genetic diagnosis (PGD) of FXS [7] and two PCR analyses (PCR screening and PCR premutation) [8]. However, it is difficult to draw a solid criterion due to different inclusive criteria, diagnostic methods, and sample sizes within each study. Although frequencies of clinical characteristics were different between ethnicities, especially in Asian and African people, which provided evidence for genetic counseling [9], FXS is still difficult to be diagnosed on the account of a lack of an obvious phenotype at birth and during prepuberty in clinic.

Previous studies have illustrated that FXS is caused by the alteration at multiple levels from mRNA shuttling to synaptic plasticity and behavioral phenotypes [10]. For example, FMRP regulated proteins in the modulation of synaptic plasticity, which maintain spine shape and dynamics [11-13]. The retardation of FMRP leads to abnormal 
group I metabotropic glutamate receptor (mGluR) signaling, together with the loss of AMPA and NMDA receptors [2], although recently clinical trials targeting on mGluR1 failed in FXR patients [14]. Specifically, the enzymes for GABA synthesis and degradation, GABA membrane transporters, and a GABA receptor scaffolding protein are downregulated in the absence of FMRP [15]. Besides, FMRP absence $\mathrm{GABA}_{\mathrm{A}}$ receptor $\alpha 1$ and $\delta$ subunits were downregulated in fmr1 gene knockout mouse and Drosophila [15-17]. All these studies suggest a perplexing, yet not well understood, link between GABAergic signaling, abnormal neuronal circuits, and dysfunctional behaviors in both FXS animal models and patients. Among all alterations of phenomenal function deficits, dendritic abnormalities are the most evident structural changes in FXS. FMRP regulates neuronal branching as well as dendritic spine morphology and density $[18,19]$. However, it remains unclear whether plastic changes of inhibitory circuits may cause abnormal spine morphology in FXS or vice versa. In this review, we summarized mechanisms on the effects of inhibitory synapse alteration from circuits to molecular interaction.

\section{Altered GABA Metabolisms in FXS Animal Models and Patients}

There have been great progresses in the altered GABA metabolism underlying FXTAS/FXS pathogenesis. Mitochondria provides energy for the cell and the brain using most of the energy among all organs. There is mounting evidence that mitochondrial dysregulation systemically contributes to the decreased cell function, even during the neonatal period of mice, first reported by Rizzo et al. [20]. It is reported that premutated hippocampal neurites contained significantly fewer mitochondria and reduced mitochondria mobility at early stage of differentiation, despite the presence of appreciable FMRP expression [21]. Together, similar significant deficits of mitochondrial dysfunction, induced by $\mathrm{Zn}$ levels, were observed in the $\mathrm{Zn}$-rich regions (the hippocampus and cerebellum of premutation carriers), with some of these effects lasting into adulthood [22, 23]. Particularly, in dysregulated GABAergic circuits, mitochondrial dysfunction plays vital role from the aspects of mitochondrial structure, number, membrane permeability, transport, fusion, and fission [21, 24-27]. It is noteworthy that abnormality of mitochondrial structure and function is regulated aberrant expression of microRNAs (miRNA) [28], while few report functions of miRNAs on GABA metabolism in Fragile X syndrome. More work should be needed to illustrate the perplexing role of deregulated miRNA expression profiles within uncommon GABA neural circuits. In a word, abnormalities of mitochondrial dysfunction induced by FMRP deficits altered GABA metabolism, contributing to the etiology of FXS/FXTAS.

In addition, glutamic acid decarboxylase (GAD) or vesicular GABA transporter protein (VGTA) and vesicular glutamate transporter protein (VGLUT) consist of two components of synaptic balance. Increased expression of VGAT relative to VGLUT expression was shown within the medial nucleus of the trapezoid body (MNTB) in FXS [29].
Their mechanisms are necessary to be further explored. In FXS patients, a reduced release of GABA from the GABAergic terminals to the presynaptic $\mathrm{GABA}_{\mathrm{B}}$ receptors might induce a decreased inhibition of neurotransmitter spillover, which conversely activated mGluR signaling [30]. One mechanism of modulating GABA release involves the synthesis and mobilization of endocannabinoids. Activation of GroupImGluRs enables mobilization of endocannabinoids in the postsynaptic neuron and negatively modulates GABA release through a mechanism known as depolarizationinduced suppression of inhibition (DSI) [31]. Therefore, in consideration of endocannabinoid mobilization in the FXS, it is reported that alterations in eCB signaling could contribute to the cognitive dysfunction associated with FXS [32]. But it only demonstrated DHPG-induced eCB-iLTD, without affecting DSI, at low concentrations. Together, relatively high concentrations of cannabinoids could affect neuropsychiatric disorders via inhibition of monoamine oxidase activity [33]. Therefore, the loss of FMRP may selectively affect specific inhibitory circuits and more evidence is needed in exploring. In the developing and mature brain, it is critical for cortical balance of excitatory and inhibitory neurons to be properly synchronized at behaviorally relevant frequencies. And thus, alteration of mGluR signaling and GABA metabolisms in this specific type of interneuron is likely to have wide-reaching effects in developing and mature cortical networks.

\section{Decreased Expression of GABA Receptor Subunits in FXS Models}

The anomalous functions of mGluR-dependent synaptic plasticity have been observed in the hippocampus of fmr1-KO mice. Activity-dependent synthesis of FMRP in maintaining forms of synaptic plasticity may be induced by augmented mGluR-LTD in hippocampal neurons [34, 35], while the initiation of long-term potentiation (LTP) is a qualitatively different functional consequence of mGluR1stimulated protein synthesis at the synapses of the hippocampus where LTD can be induced. Besides, the mGluR theory proposes that stimulation of mGluR1 induces local mRNA translation, resulting in protein synthesis that subsequently enhances the internalization of AMPA receptors [36]. This model predicts that in the absence of FMRP, the increased translation of a subset of mRNAs disturbs receptor internalization dynamics and then exaggerates internalization of AMPA receptors and weakens the synapse. Interestingly, $\mathrm{GABA}_{\mathrm{B} 1}$ and $\mathrm{GIRK}_{2}$ internalization also is reported to cause rapid and persistent weakening of $\mathrm{GABA}_{\mathrm{B}}$-activated GIRKmediated $\left(\mathrm{GABA}_{\mathrm{B}}-\mathrm{GIRK}\right)$ currents in FXS [37]. Clearly, the fate of internalized $\mathrm{GABA}_{\mathrm{A}}$ Rs will therefore play a critical role in controlling cell surface receptor levels and hence the efficacy of synaptic inhibition. This may suggest that GABA receptors take internalization process, but its underlying complicated mechanisms still need to be explored. Furthermore, FMRP absence increased steady state surface levels of $\mathrm{GABA}_{\mathrm{A}} \mathrm{Rs}$, showing a dramatic functional effect of increased surface receptor number. The mechanism underlying postendocytic $\mathrm{GABA}_{\mathrm{A}} \mathrm{R}$ sorting remains to be demonstrated, and FMRP's particular role in this process is also an area of 
active research. The impact of FMRP regulation of $\mathrm{GABA}_{\mathrm{A}} \mathrm{Rs}$ was recently shown in the hypothalamus, causing decreased food intake and loss of body weight [38]. An unresolved issue is whether FMRP acts to promote recycling of $\mathrm{GABA}_{\mathrm{A}} \mathrm{Rs}$ or prevents their lysosomal degradation.

Furthermore, different subunit combination leads to diverse expression patterns of $\mathrm{GABA}_{\mathrm{A}} \mathrm{Rs}$ at specific cell surface. Most surface receptor clusters of $\gamma 2$ receptor subunits are synaptic, while $\mathrm{GABA}_{\mathrm{A}}$ Rs containing $\alpha 5$ or $\beta 3$ subunit express higher at extrasynaptic. $\delta$ subunit is exclusively located outside the synapse at perisynaptic and extrasynaptic locations $[39,40]$. For example, it is investigated that tonic $\mathrm{GABA}_{\mathrm{A}}$ currents in the subiculum were downregulated in the fmr1 knockout mouse relative to wild-type animals [41]. These results were associated with reductions in tonic $\mathrm{GABA}_{\mathrm{A}}$ receptor subunits. Furthermore, more specific results based on the different GABAR subunits need to be expanded to better identify each function in FXS.

Results from all above pave the way for many interesting avenues of research. First, more work is needed to illustrate the molecular causes of impaired inhibition in FXS. In the Drosophila model, limited research available has demonstrated that a $\mathrm{GABA}_{\mathrm{A}}$ receptor reduction can lead to behavioral impairments. However, other research from FXS models indicated that the mechanism was likely more complicated and possibly indirect due to not only variable GABA metabolisms but also regional specificity $[40,42,43]$. For example, vision process is modulated by different GABA receptors in spread brain via tonic inhibition, such as temporal cortex, lateral geniculate nucleus (LGN) of the thalamus, and vision cortex [44, 45], while tonic inhibition is mediated via extrasynaptic $\alpha 5$ - and $\delta$-containing $\mathrm{GABA}_{\mathrm{A}}$ Rs $[40,44]$. Future research will examine that specific subunits of GABA receptor encode these vision information computations. And it is also worth noting that the role of GABA in the developing CNS is dynamic and variable between brain regions [40, 43, 46]. Another triggering idea is that impaired inhibition comes from activity-dependent synaptic plasticity alteration during developmentally critical periods [43, 47]. Both mouse and Drosophila FXS models show impaired critical period plasticity, and early activity is critical for shaping E/I synaptic balance [48-50]. These findings indicate that many mechanisms are to be explored among GABAergic neurons, GABA metabolism, and GABA receptor alteration in FXS.

\section{Impaired GABAergic Neural Circuits in FXS}

Dysfunctional mGluR1/5 signaling in excitatory synaptic circuitry has been considered as one classic mechanism underlying FXS [51-53]. But a main characteristic of the impairment is usually attributed to a failure in the inhibition of the central set or the need for a supervisory system to be involved in the inhibition of predominant manners. The increased excitability of hippocampal and neocortical circuits in FXS, due to dysregulation of glutaminergic neurons, can in turn disrupt the normal actions of inhibitory GABAergic neurons $[32,54]$. It has long been known that FXS models also display reduced function in inhibitory GABAergic circuits [55-57]. Specifically, downregulation of $\mathrm{GABA}_{\mathrm{A}}$ receptor subunits influences both the mRNA and protein levels, which would further increase the excitability of limbic and cortical circuits [39].

FMRP is widely expressed in GABAergic neurons $[58,59]$, and it is also involved in normal interneuron maturation and function modulation $[30,55,58]$. Recently, it was shown that there were lower expressions of several genes involved in GABA metabolism, including gad1, gat1, and gat4, in the brain of both mouse and Drosophila FXS models $[15,58,60]$. It is well known that GABAergic neurons can modulate neurotransmitter release in autocrine or paracrine pattern, via presynaptic $\mathrm{GABA}_{\mathrm{A}}$ and $\mathrm{GABA}_{\mathrm{B}}$ receptors [61-63]. It is indicated that dysfunctional GABAergic neurons affect balance of inhibitory/excitatory circuits particularly during early developmental critical periods, via the role of GABA attenuated regulator in FXS models [62, 64].

For now, GABAergic impairments have been reported in FXS models of Drosophila, zebrafish, and mouse. And GABAergic signaling is essential for regulating neuronal migration, maturation, and circuit formation. Therefore, defects in the GABAergic system are likely to have profound effects on neuron development and circuit work in FXS. Currently, a better understanding of early developmental changes in GABAergic system in FXS would be reckoned as the key insight into the underpinning of the FXS brain. Also, the relationship between GABAergic systems and mGluRs ones, as well as their overlapping plasticity alteration, is taken as the pivotal basement to strengthen a more comprehensive cognition of FXS.

Besides the deficits in learning and memory in these models, one consistent behavioral abnormality they share is altered circadian rhythm behaviors, which potentially mimics the sleep abnormalities seen in patients with fragile $\mathrm{X}$ syndrome. Circadian rhythm describes the approximately 24-hour cycles generated by a master pacemaker located in the suprachiasmatic nuclei (SCN) of the anterior hypothalamus of the mammalians and in the ventral lateral neurons (LNvs) of Drosophila [65]. Also, the connections between the SCN and other parts of the system are important for the control of circadian rhythms in the central nervous system [66]. Interestingly, it has been shown that the loss of FMRP and FXR2P results in arrhythmicity resulting from inappropriate neuronal communication within the central nervous system [67]. Additionally, the altered expression of the clock component has been observed in FXS animal models [67, 68]. The upregulation of FMRP increases PER1- and PER2-induced BMAL1-NPAS2 transcriptional activity, suggesting that FMRP is required for regulation of circadian behaviors. Thus, Drosophila lacking the fmrl gene exhibits altered circadian rhythms. Taken together, these results indicate that fragile X-related proteins might be associated with the induction of abnormal sleep patterns in FXS due to alterations in circadian genes; they may also play a critical role in the regulation of circadian output pathways.

Clinical studies have illustrated that melatonindependent signaling pathways can impair vigilance, learning, and memory abilities and may be linked to autistic behaviors such as abnormal anxiety responses $[69,70]$. Low melatonin 
levels are related with altered GABAergic system [71]. Furthermore, alterations in the circadian clock mechanism due to abnormal melatonin synthesis can affect the function of GABA neural circuits [70, 72]. Recently, studies using animal models of autism have indicated that clock and clock-related genes may interact in the ASD phenotype and studies using fmr $1 \mathrm{KO}$ mice have implicated clock proteins in sleep alterations in FXS [73]. Under dysfunctional FMRP conditions, GABA activity is altered by disruptions in intracellular signaling. Recent studies have proposed the existence of abnormalities in melatonin secretion and circadian patterns in individuals with FXS with ASD that are likely to be due to excessive signaling via GABA [74]. Furthermore, melatonin is helpful for treating the physical alterations of axons and dendritic spines [75, 76]. In addition, other endocrine hormones, such as oxytocin and insulin, participate GABA neuronal function via abnormal biorhythmic patterns. It is reported that oxytocin-mediated GABA excitatory-inhibitory shift during delivery is abolished in FXS model. During delivery and subsequently hippocampal neurons have elevated intracellular chloride levels and elevated gamma oscillations, which suggests the importance of oxytocin-mediated GABAergic inhibition during the process [77]. Similarly, the insulin-producing cells (IPCs) are crucial for normal insulin release and insulin-signaling in the brain and are sufficient to restore normal circadian behavior in the Drosophila FXS model [78]. Moreover, IPCs have been demonstrated to receive inputs from multiple neurotransmitters and hormones, including tachykinin, leptin, GABA, and serotonin [79]. But the specific mechanisms deserve further investigation. In brief summary, alteration of GABA inhibition is not simply linked to amplified mGluR signaling, whereas they both are regulated by circadian clock and circadian genes in depth.

Overall, current issues provide much needed in vivo evidence for GABAergic circuit impairments in FXS and set the foundation for future work linking molecular to circuit level to behavioral changes. Addressing altered GABAergic circuit function should lead to more effective treatments for FXS patients.

\section{Conclusions}

In summary, deregulated GABA metabolism, decreased expressions of GABA receptor subunits, and impaired GABAergic neural circuits contribute to abnormal behaviors in FXS. Importantly, it is noteworthy to be studied that circadian clock genes regulate substantial life activities of organism and are related to the process of growth and development in FXS models and patients. Specifically, GABA inhibition is modulated via dysfunctional biorhythmic patterns of endocrine hormones and fmrl gene. And better understanding of the GABA neural circuits will support novel therapeutic methods in FXS.

\section{Conflicts of Interest}

The authors declare that there is no conflict of interests regarding the publication of this paper.

\section{Acknowledgments}

This work was supported by the National Natural Science Foundation of China (81671081 and 31371120 to Dr. HX).

\section{References}

[1] J. D. Richter, G. J. Bassell, and E. Klann, "Dysregulation and restoration of translational homeostasis in fragile $\mathrm{X}$ syndrome," Nature Reviews Neuroscience, vol. 16, no. 10, pp. 595-605, 2015.

[2] K. Zhang, Y. J. Li, Y. Guo et al., "Elevated progranulin contributes to synaptic and learning deficit due to loss of fragile $\mathrm{X}$ mental retardation protein," Brain, vol. 140, no. 12, pp. 3215-3232, 2017.

[3] E. Chen, M. R. Sharma, X. Shi, R. K. Agrawal, and S. Joseph, "Fragile $\mathrm{X}$ mental retardation protein regulates translation by binding directly to the ribosome," Molecular Cell, vol. 54, no. 3, pp. 407-417, 2014.

[4] P. K. Todd, S. Y. Oh, A. Krans et al., "CGG repeat-associated translation mediates neurodegeneration in fragile $\mathrm{X}$ tremor ataxia syndrome," Neuron, vol. 78, no. 3, pp. 440-455, 2013.

[5] Y. T. Hwang, S. M. Aliaga, M. Arpone et al., "Partially methylated alleles, microdeletion, and tissue mosaicism in a fragile $\mathrm{X}$ male with tremor and ataxia at 30 years of age: a case report," American Journal of Medical Genetics Part A, vol. 170, no. 12, pp. 3327-3332, 2016.

[6] S. M. Aliaga, H. R. Slater, D. Francis et al., "Identification of males with cryptic Fragile $\mathrm{X}$ alleles by methylation-specific quantitative melt analysis," Clinical Chemistry, vol. 62 , no. 2 , pp. 343-352, 2016.

[7] M. Chen, M. Zhao, C. G. Lee, and S. S. Chong, "Identification of microsatellite markers $<1 \mathrm{Mb}$ from the FMR1 CGG repeat and development of a single-tube tetradecaplex PCR panel of highly polymorphic markers for preimplantation genetic diagnosis of fragile X syndrome," Genetics in Medicine, vol. 18, no. 9, pp. 869-875, 2016.

[8] A. P. Amancio, C. A. de O Melo, A. de M Vieira et al., "Molecular analysis of patients suspected of Fragile X syndrome," Genetics and Molecular Research, vol. 14, no. 4, pp. 1466014669, 2015.

[9] C. Charalsawadi, J. Wirojanan, S. Jaruratanasirikul, N. Ruangdaraganon, A. Geater, and P. Limprasert, "Common clinical characteristics and rare medical problems of Fragile $\mathrm{X}$ syndrome in Thai patients and review of the literature," International Journal of Pediatrics, vol. 2017, Article ID 9318346, 11 pages, 2017.

[10] T. Yang, H. Zhao, C. Lu et al., "Synaptic plasticity, a prominent contributor to the anxiety in Fragile X syndrome," Neural Plasticity, vol. 2016, Article ID 9353929, 12 pages, 2016.

[11] T. L. Schmit, J. A. Dowell, M. E. Maes, and M. Wilhelm, "c-Jun $\mathrm{N}$-terminal kinase regulates mGluR-dependent expression of post-synaptic FMRP target proteins," Journal of Neurochemistry, vol. 127, no. 6, pp. 772-781, 2013.

[12] F. Niere, J. R. Wilkerson, and K. M. Huber, "Evidence for a fragile $\mathrm{X}$ mental retardation protein-mediated translational switch in metabotropic glutamate receptor-triggered arc translation and long-term depression," The Journal of Neuroscience, vol. 32, no. 17, pp. 5924-5936, 2012.

[13] J. Steinberg and C. Webber, "The roles of FMRP-regulated genes in autism spectrum disorder: single- and multiple-hit 
genetic etiologies," American Journal of Human Genetics, vol. 93, no. 5, pp. 825-839, 2013.

[14] A. Mullard, "Fragile X disappointments upset autism ambitions," Nature Reviews Drug Discovery, vol. 14, no. 3, pp. 151-3, 2015.

[15] C. L. Gatto, D. Pereira, and K. Broadie, "GABAergic circuit dysfunction in the Drosophila Fragile X syndrome model," Neurobiology of Disease, vol. 65, pp. 142-159, 2014.

[16] V. Sabanov, S. Braat, L. D'Andrea et al., "Impaired GABAergic inhibition in the hippocampus of Fmrl knockout mice," Neuropharmacology, vol. 116, pp. 71-81, 2017.

[17] S. Braat, C. D'Hulst, I. Heulens et al., "The GABAA receptor is an FMRP target with therapeutic potential in fragile $\mathrm{X}$ syndrome," Cell Cycle, vol. 14, no. 18, pp. 2985-2995, 2015.

[18] S. Scotto-Lomassese, A. Nissant, T. Mota et al., "Fragile X mental retardation protein regulates new neuron differentiation in the adult olfactory bulb," The Journal of Neuroscience, vol. 31, no. 6, pp. 2205-2215, 2011.

[19] A. Khayachi, C. Gwizdek, G. Poupon et al., "Sumoylation regulates FMRP-mediated dendritic spine elimination and maturation," Nature Communications, vol. 9, no. 1, p. 757, 2018.

[20] G. Rizzo, F. Pizza, C. Scaglione et al., "A case of fragile X premutation tremor/ataxia syndrome with evidence of mitochondrial dysfunction," Movement Disorders, vol. 21, no. 9, pp. 1541-1542, 2006.

[21] Z. Cao, S. Hulsizer, Y. Cui et al., "Enhanced asynchronous $\mathrm{Ca}^{2+}$ oscillations associated with impaired glutamate transport in cortical astrocytes expressing Fmrl gene premutation expansion," Journal of Biological Chemistry, vol. 288, no. 19, pp. 13831-13841, 2013.

[22] E. Napoli, C. Ross-Inta, S. Wong et al., "Altered zinc transport disrupts mitochondrial protein processing/import in fragile $\mathrm{X}$-associated tremor/ataxia syndrome," Human Molecular Genetics, vol. 20, no. 15, pp. 3079-3092, 2011.

[23] E. Napoli, C. Ross-Inta, G. Song et al., "Premutation in the Fragile X mental retardation 1 (FMR1) gene affects maternal Zn-milk and perinatal brain bioenergetics and scaffolding," Frontiers in Neuroscience, vol. 10, p. 159, 2016.

[24] R. Hagerman, J. Au, and P. Hagerman, "FMR1 premutation and full mutation molecular mechanisms related to autism," Journal of Neurodevelopmental Disorders, vol. 3, no. 3, pp. 211-224, 2011.

[25] R. Hagerman, G. Hoem, and P. Hagerman, "Fragile X and autism: intertwined at the molecular level leading to targeted treatments," Molecular Autism, vol. 1, no. 1, p. 12, 2010.

[26] E. S. Kaplan, Z. Cao, S. Hulsizer et al., "Early mitochondrial abnormalities in hippocampal neurons cultured from Fmr1 pre-mutation mouse model," Journal of Neurochemistry, vol. 123, no. 4, pp. 613-621, 2012.

[27] A. Yao, S. Jin, X. Li et al., "Drosophila FMRP regulates microtubule network formation and axonal transport of mitochondria," Human Molecular Genetics, vol. 20, no. 1, pp. 51-63, 2011.

[28] R. Richter-Dennerlein, S. Dennerlein, and P. Rehling, "Integrating mitochondrial translation into the cellular context," Nature Reviews Molecular Cell Biology, vol. 16, no. 10, pp. 586-592, 2015.

[29] S. E. Rotschafer, S. Marshak, and K. S. Cramer, "Deletion of Fmrl alters function and synaptic inputs in the auditory brainstem," PLoS One, vol. 10, no. 2, article e0117266, 2015.
[30] M. D. Lange, K. Jüngling, L. Paulukat et al., "Glutamic acid decarboxylase 65: a link between GABAergic synaptic plasticity in the lateral amygdala and conditioned fear generalization," Neuropsychopharmacology, vol. 39, no. 9, pp. 2211-2220, 2014.

[31] N. Varma, G. C. Carlson, C. Ledent, and B. E. Alger, "Metabotropic glutamate receptors drive the endocannabinoid system in hippocampus," The Journal of Neuroscience, vol. 21, no. 24, article Rc188, 2001.

[32] L. Zhang and B. E. Alger, "Enhanced endocannabinoid signaling elevates neuronal excitability in fragile X syndrome," The Journal of Neuroscience, vol. 30, no. 16, pp. 5724-5729, 2010.

[33] Z. Fisar, "Inhibition of monoamine oxidase activity by cannabinoids," Naunyn-Schmiedeberg's Archives of Pharmacology, vol. 381, no. 6, pp. 563-572, 2010.

[34] J. Zhang, L. Hou, E. Klann, and D. L. Nelson, “Altered hippocampal synaptic plasticity in the FMR1 gene family knockout mouse models," Journal of Neurophysiology, vol. 101, no. 5, pp. 2572-2580, 2009.

[35] A. J. Iliff, A. J. Renoux, A. Krans, K. Usdin, M. A. Sutton, and P. K. Todd, "Impaired activity-dependent FMRP translation and enhanced mGluR-dependent LTD in Fragile X premutation mice," Human Molecular Genetics, vol. 22, no. 6, pp. 1180-1192, 2013.

[36] H. Wang, S. S. Kim, and M. Zhuo, "Roles of fragile X mental retardation protein in dopaminergic stimulationinduced synapse-associated protein synthesis and subsequent $\alpha$-amino-3-hydroxyl-5-methyl-4-isoxazole-4-propionate (AMPA) receptor internalization," Journal of Biological Chemistry, vol. 285, no. 28, pp. 21888-21901, 2010.

[37] S. Lecca, A. Pelosi, A. Tchenio et al., "Rescue of $G_{A B A}$ and GIRK function in the lateral habenula by protein phosphatase $2 \mathrm{~A}$ inhibition ameliorates depression-like phenotypes in mice," Nature Medicine, vol. 22, no. 3, pp. 254-261, 2016.

[38] B. Liu, L. Li, J. Chen, Z. Wang, Z. Li, and Q. Wan, "Regulation of GABAA receptors by fragile X mental retardation protein," International Journal of Physiology, Pathophysiology and Pharmacology, vol. 5, no. 3, pp. 169-176, 2013.

[39] M. Drexel, E. Kirchmair, and G. Sperk, "Changes in the expression of $\mathrm{GABA}_{\mathrm{A}}$ receptor subunit mRNAs in parahippocampal areas after kainic acid induced seizures," Frontiers in Neural Circuits, vol. 7, p. 142, 2013.

[40] G. Curia, T. Papouin, P. Seguela, and M. Avoli, "Downregulation of tonic GABAergic inhibition in a mouse model of fragile X syndrome," Cerebral Cortex, vol. 19, no. 7, pp. 1515-1520, 2009.

[41] N. Zhang, Z. Peng, X. Tong et al., "Decreased surface expression of the $\delta$ subunit of the $\mathrm{GABA}_{\mathrm{A}}$ receptor contributes to reduced tonic inhibition in dentate granule cells in a mouse model of fragile X syndrome," Experimental Neurology, vol. 297, pp. 168-178, 2017.

[42] C. D'Hulst, I. Heulens, N. van der Aa et al., "Positron emission tomography (PET) quantification of $\mathrm{GABA}_{\mathrm{A}}$ receptors in the brain of Fragile X patients," PLoS One, vol. 10, no. 7, article e0131486, 2015.

[43] S. Kratovac and J. G. Corbin, "Developmental changes in expression of inhibitory neuronal proteins in the Fragile $\mathrm{X}$ syndrome mouse basolateral amygdala," Brain Research, vol. 1537, pp. 69-78, 2013.

[44] P. H. Frederikse, A. Nandanoor, and C. Kasinathan, "Fragile X syndrome FMRP co-localizes with regulatory targets PSD-95, 
GABA receptors, CaMKII $\alpha$, and mGluR5 at fiber cell membranes in the eye lens," Neurochemical Research, vol. 40, no. 11, pp. 2167-2176, 2015.

[45] J. A. Hirsch, X. Wang, F. T. Sommer, and L. M. Martinez, "How inhibitory circuits in the thalamus serve vision," Annual Review of Neuroscience, vol. 38, no. 1, pp. 309-329, 2015.

[46] J. A. C. Broek, Z. Lin, H. M. de Gruiter et al., "Synaptic vesicle dynamic changes in a model of fragile X," Molecular Autism, vol. 7, no. 1, p. 17, 2016.

[47] C. A. Doll and K. Broadie, "Activity-dependent FMRP requirements in development of the neural circuitry of learning and memory," Development, vol. 142, no. 7, pp. 1346-1356, 2015.

[48] C. A. Doll and K. Broadie, "Impaired activity-dependent neural circuit assembly and refinement in autism spectrum disorder genetic models," Frontiers in Cellular Neuroscience, vol. 8, p. 30, 2014.

[49] B. S. Martin, J. G. Corbin, and M. M. Huntsman, "Deficient tonic GABAergic conductance and synaptic balance in the fragile X syndrome amygdala," Journal of Neurophysiology, vol. 112, no. 4, pp. 890-902, 2014.

[50] C. J. Westmark, S. C. Chuang, S. A. Hays et al., "APP causes hyperexcitability in Fragile X mice," Frontiers in Molecular Neuroscience, vol. 9, p. 147, 2016.

[51] S.-C. Mao, C.-H. Chang, C.-C. Wu, M. J. Orejanera, O. J. Manzoni, and P.-W. Gean, "Inhibition of spontaneous recovery of fear by mGluR5 after prolonged extinction training," PLoS One, vol. 8, no. 3, article e59580, 2013.

[52] A. Michalon, A. Bruns, C. Risterucci et al., "Chronic metabotropic glutamate receptor 5 inhibition corrects local alterations of brain activity and improves cognitive performance in fragile X mice," Biological Psychiatry, vol. 75, no. 3, pp. 189-197, 2014.

[53] A. S. Pop, B. Gomez-Mancilla, G. Neri, R. Willemsen, and F. Gasparini, "Fragile X syndrome: a preclinical review on metabotropic glutamate receptor 5 (mGluR5) antagonists and drug development," Psychopharmacology, vol. 231, no. 6, pp. 1217-1226, 2014.

[54] A. Straiker, K. T. Min, and K. Mackie, "Fmr1 deletion enhances and ultimately desensitizes $\mathrm{CB}_{1}$ signaling in autaptic hippocampal neurons," Neurobiology of Disease, vol. 56, pp. 1-5, 2013.

[55] T. Nomura, T. F. Musial, J. J. Marshall et al., "Delayed maturation of fast-spiking interneurons is rectified by activation of the TrkB receptor in the mouse model of Fragile X syndrome," The Journal of Neuroscience, vol. 37, no. 47, pp. 11298-11310, 2017.

[56] K. Ruby, K. Falvey, and R. J. Kulesza, “Abnormal neuronal morphology and neurochemistry in the auditory brainstem of Fmr1 knockout rats," Neuroscience, vol. 303, pp. 285-298, 2015.

[57] L. M. Franco, Z. Okray, G. A. Linneweber, B. A. Hassan, and E. Yaksi, "Reduced lateral inhibition impairs olfactory computations and behaviors in a Drosophila model of fragile X syndrome," Current Biology, vol. 27, no. 8, pp. 1111-1123, 2017.

[58] J. L. Olmos-Serrano, S. M. Paluszkiewicz, B. S. Martin, W. E. Kaufmann, J. G. Corbin, and M. M. Huntsman, "Defective GABAergic neurotransmission and pharmacological rescue of neuronal hyperexcitability in the amygdala in a mouse model of fragile X syndrome," The Journal of Neuroscience, vol. 30, no. 29, pp. 9929-9938, 2010.
[59] S. B. Christie, M. R. Akins, J. E. Schwob, and J. R. Fallon, "The FXG: a presynaptic fragile $\mathrm{X}$ granule expressed in a subset of developing brain circuits," The Journal of Neuroscience, vol. 29, no. 5, pp. 1514-1524, 2009.

[60] A. El Idrissi, X. H. Ding, J. Scalia, E. Trenkner, W. T. Brown, and C. Dobkin, "Decreased $\mathrm{GABA}_{\mathrm{A}}$ receptor expression in the seizure-prone fragile $\mathrm{X}$ mouse," Neuroscience Letters, vol. 377, no. 3, pp. 141-146, 2005.

[61] F. F. Trigo, A. Marty, and B. M. Stell, "Axonal GABA receptors," The European Journal of Neuroscience, vol. 28, no. 5, pp. 841-848, 2008.

[62] D. C. Adusei, L. K. K. Pacey, D. Chen, and D. R. Hampson, "Early developmental alterations in GABAergic protein expression in fragile X knockout mice," Neuropharmacology, vol. 59, no. 3, pp. 167-171, 2010.

[63] C. D'Hulst, I. Heulens, J. R. Brouwer et al., "Expression of the GABAergic system in animal models for fragile X syndrome and fragile X associated tremor/ataxia syndrome (FXTAS)," Brain Research, vol. 1253, pp. 176-183, 2009.

[64] J. Y. Kang, J. Chadchankar, T. N. Vien et al., "Deficits in the activity of presynaptic $\gamma$-aminobutyric acid type B receptors contribute to altered neuronal excitability in fragile X syndrome," The Journal of Biological Chemistry, vol. 292, no. 16, pp. 6621-6632, 2017.

[65] N. I. Muraro, N. Pirez, and M. F. Ceriani, "The circadian system: plasticity at many levels," Neuroscience, vol. 247, pp. 280-293, 2013.

[66] W. J. Schwartz, R. A. Gross, and M. T. Morton, "The suprachiasmatic nuclei contain a tetrodotoxin-resistant circadian pacemaker," Proceedings of the National Academy of Sciences of the United States of America, vol. 84, no. 6, pp. 1694-1698, 1987.

[67] J. Zhang, Z. Fang, C. Jud et al., "Fragile X-related proteins regulate mammalian circadian behavioral rhythms," American Journal of Human Genetics, vol. 83, no. 1, pp. 43-52, 2008.

[68] S. Xu, M. Poidevin, E. Han, J. Bi, and P. Jin, "Circadian rhythm-dependent alterations of gene expression in Drosophila brain lacking fragile X mental retardation protein," PLoS One, vol. 7, no. 5, article e37937, 2012.

[69] G. Kulman, P. Lissoni, F. Rovelli, M. G. Roselli, F. Brivio, and P. Sequeri, "Evidence of pineal endocrine hypofunction in autistic children," Neuro Endocrinology Letters, vol. 21, no. 1, pp. 31-34, 2000.

[70] J. Won, Y. Jin, J. Choi et al., "Melatonin as a novel interventional candidate for Fragile $\mathrm{X}$ syndrome with autism spectrum disorder in humans," International Journal of Molecular Sciences, vol. 18, no. 6, 2017.

[71] D. Hodge, T. M. Carollo, M. Lewin, C. D. Hoffman, and D. P. Sweeney, "Sleep patterns in children with and without autism spectrum disorders: developmental comparisons," Research in Developmental Disabilities, vol. 35, no. 7, pp. 1631-1638, 2014.

[72] G. J. Bassell and S. T. Warren, "Fragile X syndrome: loss of local mRNA regulation alters synaptic development and function," Neuron, vol. 60, no. 2, pp. 201-214, 2008.

[73] E. G. Bechara, M. C. Didiot, M. Melko et al., "A novel function for fragile $\mathrm{X}$ mental retardation protein in translational activation," PLoS Biology, vol. 7, no. 1, article e16, 2009.

[74] A. Miranda-Paez, S. R. Zamudio, P. Vazquez-Leon, V. Sandoval-Herrera, I. Villanueva-Becerril, and G. Carli, "Effect of melatonin injection into the periaqueductal gray on antinociception and tonic immobility in male rats," Hormones and Behavior, vol. 89, pp. 23-29, 2017. 
[75] T. Ikeno and R. J. Nelson, "Acute melatonin treatment alters dendritic morphology and circadian clock gene expression in the hippocampus of Siberian hamsters," Hippocampus, vol. 25, no. 2, pp. 142-148, 2015.

[76] L. Mendoza-Viveros, C. K. Chiang, J. L. K. Ong et al., "miR-132/212 modulates seasonal adaptation and dendritic morphology of the central circadian clock," Cell Reports, vol. 19, no. 3, pp. 505-520, 2017.

[77] R. Tyzio, R. Nardou, D. C. Ferrari et al., "Oxytocin-mediated GABA inhibition during delivery attenuates autism pathogenesis in rodent offspring," Science, vol. 343 , no. 6171 , pp. $675-$ 679, 2014.

[78] R. E. Monyak, D. Emerson, B. P. Schoenfeld et al., "Insulin signaling misregulation underlies circadian and cognitive deficits in a Drosophila fragile X model," Molecular Psychiatry, vol. 22, no. 8, pp. 1140-1148, 2017.

[79] D. R. Nässel, O. I. Kubrak, Y. Liu, J. Luo, and O. V. Lushchak, "Factors that regulate insulin producing cells and their output in Drosophila," Frontiers in Physiology, vol. 4, p. 252, 2013. 


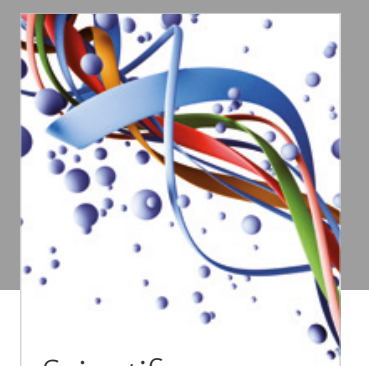

Scientifica
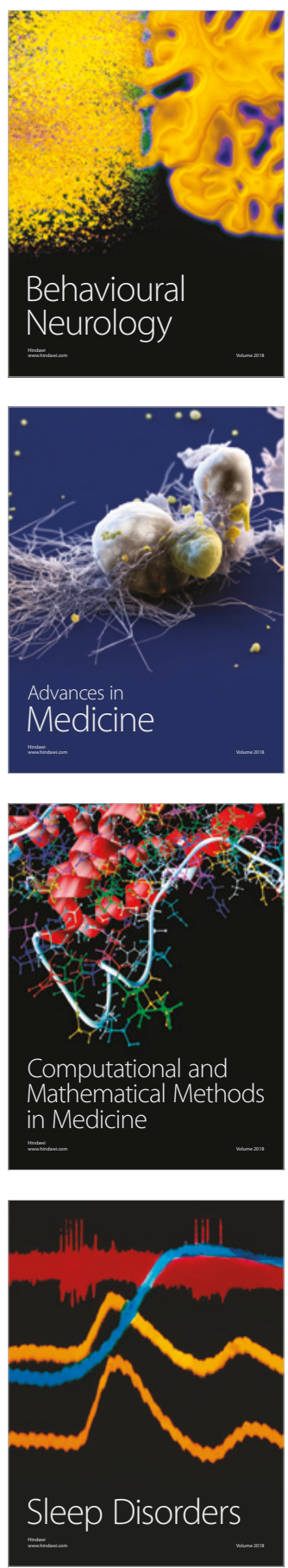

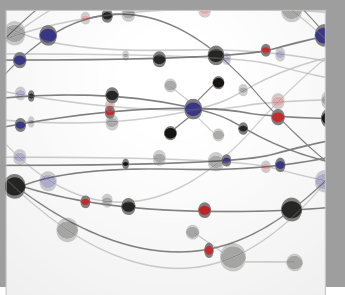

The Scientific World Journal

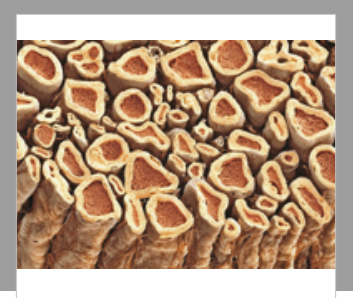

Case Reports in

Neurological Medicine

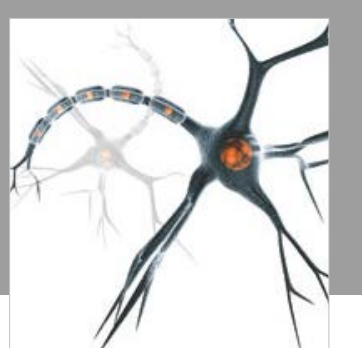

Neural Plasticity

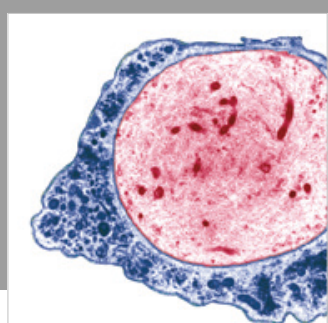

Multiple Sclerosis

International

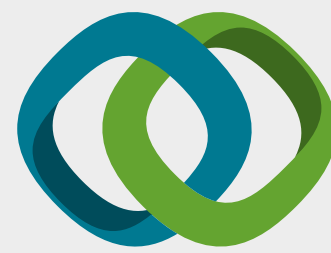

Hindawi

Submit your manuscripts at

www.hindawi.com
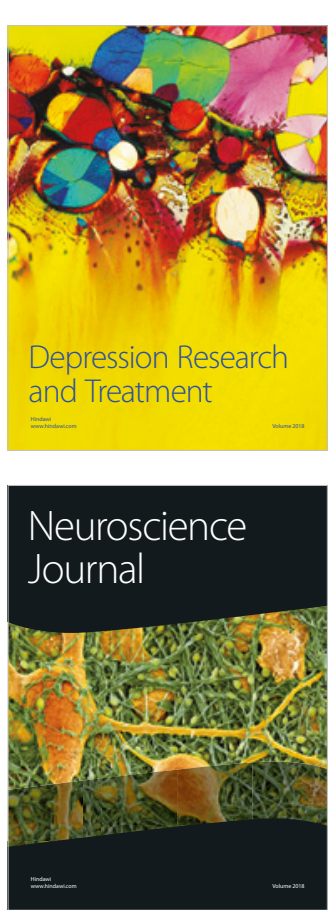

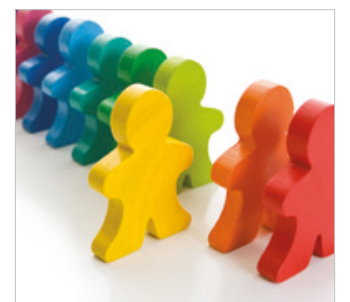

Autism

Research and Treatment
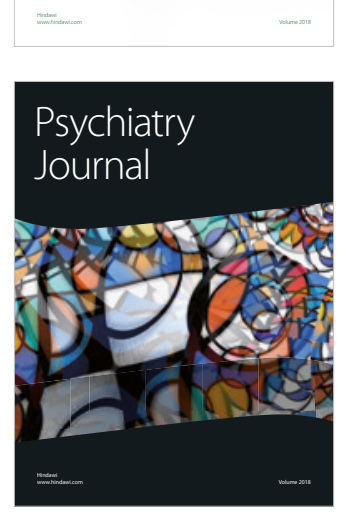
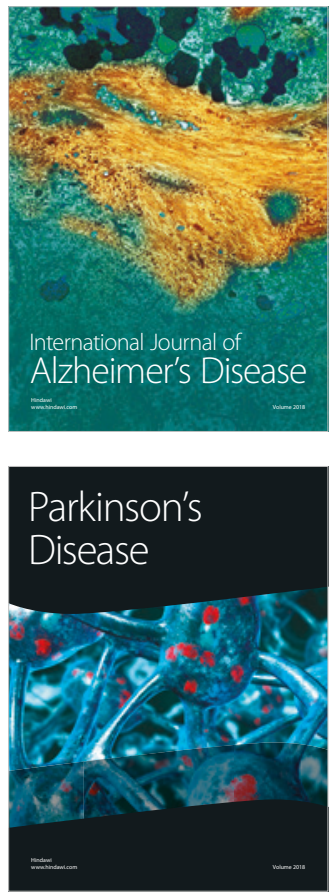
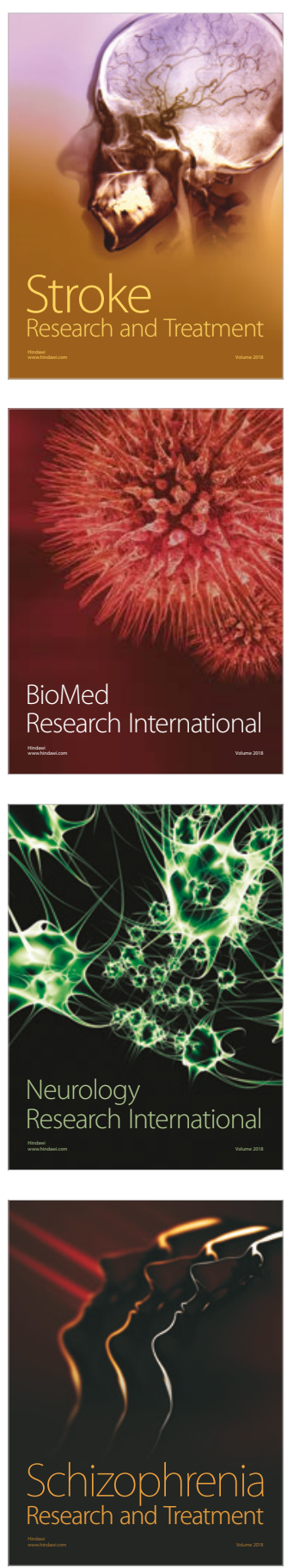